It is a great pity that our classification must be mechanical. The mathematics of the subject, as Dr. Wilbur says, very clearly show that we can only have a certain number of places for distribution of the causes of death. That necessitates the absolute loss, from certain columns of mortality, of an indefinite number of causes leading to death, and perhaps introduces inevitable error, since we who are the statisticians compiling these data are rather impressed with our own side of the question of assigning position to certificates of death. It was an excellent idea in the Census Bureau to address inquiries to a great many men in the leading specialties in medicine, asking them to express opinions or give definitions of the various titles in the international classification. I think in that way we can eliminate our own particular bias; or, if we can not eliminate it, we can minimize its error so as to make it of little practical importance.

I received one of these inquiry sheets, of which this is a printed copy, is it not, Dr. Wilbur?

DR. WILBUR-No; that is an average rating from a cudy of a number of those sheets, representing the average opinion of a certain number of statisticians and sanitarians; merely a preliminary study for the purposes of this meeting.

DR. FuLToN $-I$ received such a sheet, asking me to give a rating to each of the titles. I went into it with care, as a man interested in the subject and as a specialist. After I was done $I$ found $m y$ mind in exactly the condition in which Dr. Wilbur tells us the minds of so many medical men of the country are. After going over the entire list and reviewing it I found much in my own notes to criticise. Desiring to satisfy myself, I drew a second column, and put down an alternate series of ratings, and $I$ found that in all important titles - the very definite diseases-I had no inclination to change the rating at all. There were a number of titles as to which $I$ was inclined to very slightly increase or diminish my first rating. There were a considerable number of .rather indefinite titles to which, on second consideration, I gave a rating very different from that which $I$ had given at first. $I$ was greatly interested in hearing Dr. Wilbur say that such was about the state of mind of other men.

I confess that I don't -understand how Dr. Wilbur is going to make this mathematical basis of assignment available for clerks. I am inclined to believe that he will work it out in some practical way, although at present, as I say, I don't understand it. What I do understand very clearly, however, is that this work is going to be available for a successful revision of our classification every ten years. Such careful study year after year will enable us to take very definite and complete data to the next decennial revision. For this purpose I feel assured that the great practical utility is coming out of this part of the work; for the other I am hopeful, though I confess $I$ don't understand it at present.

Dr. G. T. Swarts, Providence, R. I.-My feelings are precisely those stated by Dr. Fulton. When I commenced to ex press my opinion in figures in regard to the comparative valua tion of these different diseases, $I$ found myself somewhat in a quandary. After making my rating the question was whether it was satisfactory to me. I think the result will be that we will have to take the average, as was done in this acceptance of nomenclature classification. I am not satisfied with the classification at all. There are a great many subjects of im. portance left out, and a great many causes I don't agree with. Nevertheless, I am willing to let my personal opinion go, so that when the statistician finds some reports from one state and some from another, he will know that they are all classified from the same standpoint.

I am willing to submit my figures to what the Census Bureau finds is a proper average. Whether it will prove sat isfactory or not, seems ambiguous to me at present, but surely, in the course of ten years, we ought to get to some point where we can understand the subject. It must be, however, thoroughly threshed out in the meanwhile.

Salt and Molasses for Cancer.-Salt as a cure for cancer is announced from India in the lav press, and an Australian physician has found molasses to be a sure cure. Salt and molasses have, at least, the valuable points of cheapness and simplicity.

\section{THE PROPER PERINEAL PROSTATECTOMY INCISION.}

NICHOLAS SENN, M.D., LL.D.

Professor of Surgery Rush Medical College; Surgeon-in-Chlef st. Joseph's Hospital; Attending Surgeon Presbyterian Hospltal.

\section{CHICAgo.}

Prostatectomy is the operation which is now attracting much attention among surgeons here and abroad. The more general adoption of this operation is comparatively of recent date. The necessity of the removal of the enlarged prostate gland, the most frequent cause of urinary obstruction in men advanced in years, has been recognized for a long time, but it was not until recently that the technic of the operation has been perfected to a degree that rendered it sufficiently safe to bring it within the scope of feasible and advisable surgical procedures. The literature on this subject is growing with astonishing rapidity. Individual and collective experiences are being published in the medical press at short intervals in bringing to the notice of the profession the different operative procedures which are being devised and practiced. The question as to the most feasible route by which to attach the diseased prostate has not been definitely settled. The suprapubic method has many weighty advocates, and for a time was deemed the easiest, most efficient and safest. The perineal route has, however, been given a more extended trial, and a very large experience appears to have decided in its favor. From an anatomic standpoint the perineal operation is certainly the most rational of the two, and will undoubtedly survive the test of time. The removal of the enlarged prostate does not always meet all the indications in the case operated on. Few cases come to the surgeon in which the bladder is intact in consequence of the mechanical obstruction or infection, hence in the majority of cases it becomes necessary to establish free drainage after the removal of the prostate for the purpose of initiating a successful treatment for the coexisting complications. In making the external incision the surgeon must therefore have in view the exposure of the prostate to sight and touch as freely as can be done with safety, and to provide for free drainage of the bladder and the perineal wound. The semilunar incision of Zuckerkandl and the triangular incision of Kocher accomplish neither of these objects to the fullest extent. I have given both of them a fair test and have become familiar with their of them a fair test and have become familiar with the difficulty encountered in establishing free and efficient drainage for the bladder and perineal wound. The triangular incision of Kocher circumscribes a long wedgeshaped flap, the apex of which is liable to slough, and the curved incision of Zuckerkandl does not procure the necessary amount of room through which the prostate, in difficult cases, can be remored with the requisite degree of precision and safety, and both incisions leave a wound difficult to drain efficiently. The median incision so much praised by some surgeons is altogether inadequate in difficult cases. Every surgeon who has had an extensive experience in perineal prostatectomy has learned that not all enlarged prostates can be removed by enucleation. There are cases in which the diseased organ must be removed by morcellement. It is a rule that surgery which it is always well to bear in mind, and that is to operate as little as possible in the dark in importh ant anatomic localities, and this rule applies with special force to perineal prostatectomy. In obese subjectand in cases of very large prostates it is often excedin ingly difficult to bring the parts to be removed withi 
reach of the index finger. It is under these trying circumstances that the operator will appreciate the advantages of an incision that will expose the prostate freely and bring it within easy reach of the finger or the instruments required for its removal. Very little is gained by attempts to render the prostate more accessible by intravesical instrumental pressure, and such efforts are by no means always harmless. The danger from

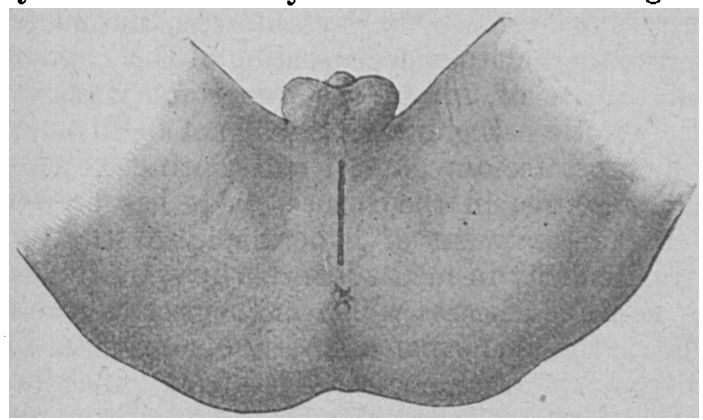

Fig. 1.- Median perineal Incision.

hemorrhage and accidental wounding of the peritoneum is reduced to a minimum by resorting to an incision that will expose the prostate in the freest possible manner to sight and touch. I have attempted to do this by combining the median incision with two lateral incisions representing in outline an inverted capital Y. The median incision is made in the usual way, laying bare the membranous portion of the urethra. The lateral in-

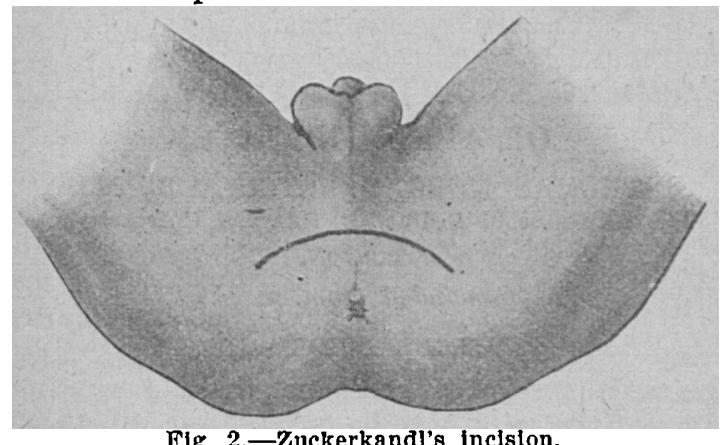

cisions are carried from the lower angle of the median to a point half way between the anal margin and the tuberosity of the ischium, cutting through about the same structures as are involved in the lateral operation for stone in the bladder. The wound is next deepened largely by the use of blunt instruments and all hemorrhage arrested as it occurs, maintaining throughout the

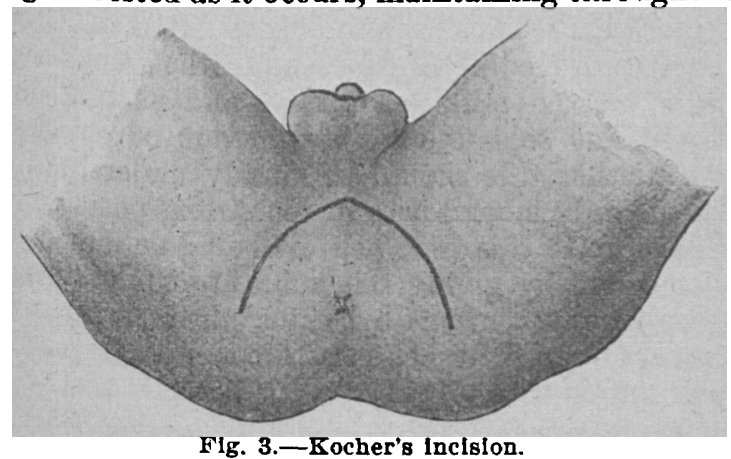

entire operation practically a bloodless field. This will give the operator an opportunity to recognize and identify the tissues as he proceeds with the dissection. In this comparatively bloodless way the rectum is detached until the membranous portion of the urethra and the lower segment of the prostate can be distinctly seen and felt in the apex of the deep triangular wound. By using narrow flat deep retractors the rectum is pushed backward and the wound margins are retracted laterally, thus exposing freely the parts concerned in the next step of the operation. I then proceed as follows: On a grooved staff which is now inserted into the bladder the membranous portion of the urethra is incised and the grooved director of Wheelhouse inserted into the bladder. Withdrawing the staff and using the director as

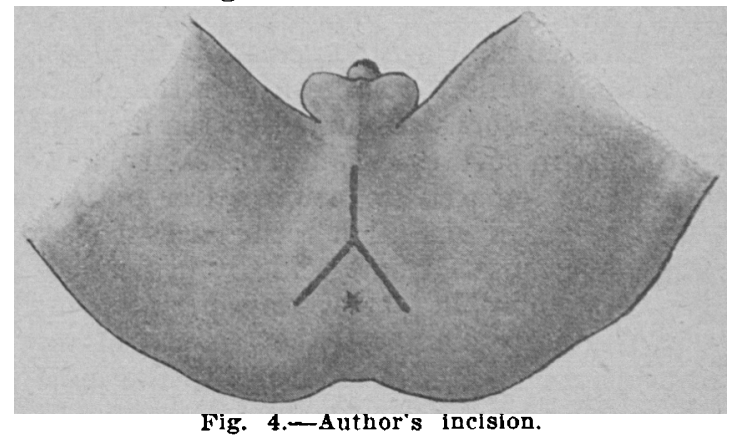

a guide the prostatic part of the urethra is dilated with the left index finger which, after it enters the bladder, serves as a blunt hook with which the prostate is drawn gently downward and forward into the wound. The capsule of the gland is next incised transversely and with the opposite index finger enucleation of the left lobe is commenced. This part of the operation is usually easy,

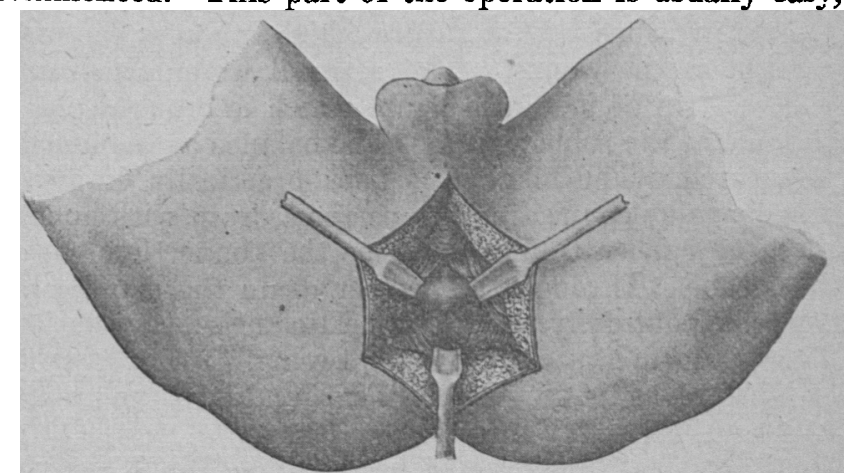

Fig. 5.-Prostate and membranous portion of urethra seen on re traction of wound margins.

sometimes difficult and not infrequently impossible. Recklessness and undue violence are to be carefully

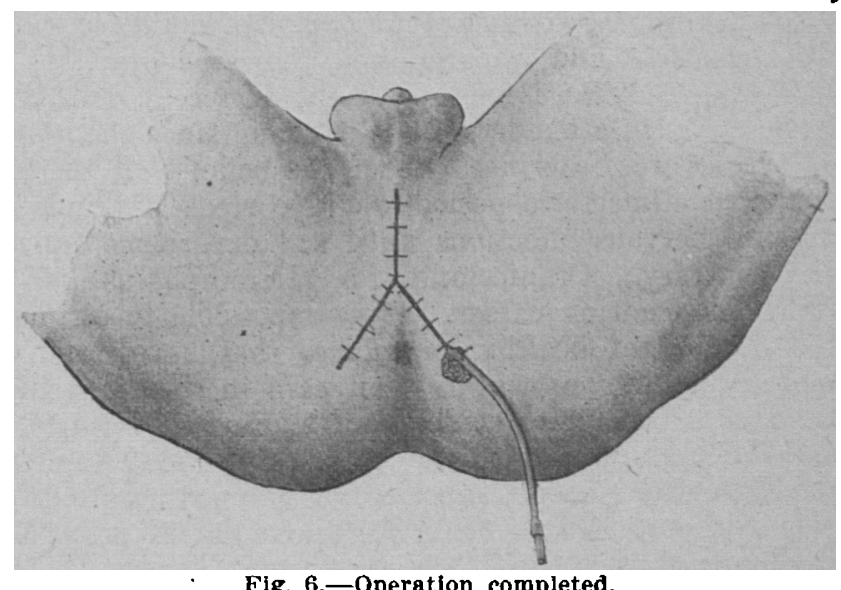

avoided. Enucleation is often facilitated by grasping the lower part of the prostate with my bullet forceps or some other grasping instrument on which traction is made during the process of enucleation. I seldom attempt to remove the prostate in its entirety. Occasionally this can be done, but usually it will be found much easier to remove only one lobe at a time. After the 
removal of the left lobe the right index finger is inserted into the bladder and the right lobe enucleated with the left. If it is found impracticable to remove the prostate by enucleation morcellement must be resorted to. The finger in the bladder is almost indispensable in operating by this method. With it the parts are brought within easier reach and it serves at the same time as a valuable guide for the use of the cutting and traction instruments. With grasping forceps portions of the gland are seized, when the necessary traction is made by an assistant while the surgeon does the cutting with blunt-pointed scissors well curved on the flat. Complete prostatectomy in such cases is not necessary, but enough tisstic must be remored to insure a free outlet for the urine and to guard against a recurrence of obstruction from the same cause. I am decidedly in favor of a preliminary cystotomy in performing perineal prostatectomy, as it greatly facilitates the removal of the gland by enucleation or morcellement, and in the majority of cases it becomes a necessity for the treatment of complicating affections of the bladder. It is preferable to incise the urethra and take advantage of such an opening into the bladder during the operation than to tear it accidentally, as is so often done when operators undertake a perineal prostatectomy without a preliminary cystotomy. I invariably drain the bladder by inserting a soft rubber drain with two oval fenestra near the vesical end. The drain is fastened in the lower angle of one of the lateral incisions with a suture which includes the outer margin of the wound. The perineal wound is drained with a strip of iodoform gauze which is brought out on the side of the rubber drain. The balance of the incision is sutured. The bladder is kept practically empty by siphonage by connecting the perineal drain with another piece of rubber tubing, making the connection with a glass tube. Through this rubber drain the bladder can be washed out daily with appropriate antiseptic solutions. The iodoform gauze drain should remain for at least five or six days, as its presence in the wound is of the greatest value in preventing infection by leakage of septic urine. The bladder drainage must be continued until the condition of the urine is such as to warrant suspension of intravesical medication. In regard to the indications for prostatectomy history will repeat itself. Every new surgical procedure has had its enthusiastic advocates who championed extreme riews and who would recognize no exceptions, and who followed a routine practice. I will refer only to a few operations of recent date that have had such a history. It is not long since thousands of ovaries were sacrificed under the belief that the anticipated climacteric period would correct all kinds of obscure nervous affections until sad experience proved the contrary. Craniectomy for microcephalus had a short but exciting career. It is impossible to estimate the number of healthy appendices that have been removed by surgeons who rely on pain in the right iliac fossa as a conclusive evidence of a diseased vermiform appendix, and resort to the knife as the only remedy. Castration for enlarged prostate has unsexed hundreds of men without yielding them an equivalent in relief for the loss sustained. Gall-bladder surgery has now a few extremists who affirm that it should be dealt with in the same medical manner as the appendix when it is the seat of disease. Instances could be multiplied, but the early history of the operations mentioned will suffice that new operations are very likely to be abused when first placed on trial, and prostatectomy can not escape a similar fate. It is impossible at the present time, so early in the history of this operation, to formulate the exact indications. The size of the prostate is no criterion to go by in deciding on the propriety or necessity of an operation as a large prostate may cause less disturbance of function of the bladder than one only moderately enlarged. The degree of obstruction depends more on the location of the enlargement and the effect of pressure on the urethra than the size of the swelling. Every prudent surgeon would hesitate to recommend a prostatectomy in men 70 to 80 years of age, the subjects of extensive atheromatous degeneration of the arteries or of organic disease of any of the important viscera. Systematic aseptic catheterization will not be displaced entirely by prostatectomy, and it will continue to be a most valuable resource in the future, as it has been in the past, in the treatment of hypertrophy of the prostate, more particularly in men advanced in years the subjects of degenerative diseases which in themselves would contraindicate the performance of any operation of importance except as an immediate life-saving procedure. It will be with this as with any other operative procedure that the best results will be obtained by surgeons who search as carefully for the negative as for the positive indications for the operation. The present technic of prostatectomy will be greatly improved, and its legitimate range of usefulness will be widened with increasing clinical experience and with a more definite knowledge of the etiology and pathology of senile hypertrophy of this gland.

\section{THE ESSENTIAL PRINCIPLES OF INFANT FEEDING AND THE MODERN METHODS OF APPLYING THEM.}

\author{
IHOMAS MORGAN ROTCH, A.B., M.D. \\ Professor of Pedlatries, Harvard University. \\ BOSTON.
}

(Concluded from p. 35.)

\section{MILK LABORATORIES.}

The next question is where and in what way the modification of the milk is to be carried out. This problem has been to a great extent solved since the establishment of what are termed milk laboratories. Up to the present time the milk laboratories have been under the control of one company called The Waker-Gordon Laboratory Company, which was established in Boston in 1891 by Mr. G. E. Gordon, Mr. Geo. H. Walker and Mr. J. H. Waterhouse. These milk laboratories are intended to take the same position toward the modification of milk that apothecary shops have to drugs. That is, directions for substitute feeding can be sent in the form of a prescription to the milk laboratory, and should only be cription to the milk laboratory, and should only.ian. dispensed and sold under the direction of.a physicia it It is well that this should be clearly understood, as ich places the milk laboratories in the strong position which they have up to this time held by being endorsed by the physician and by giving the public to understand that they are merely a means by which physicians can accurately carry out their ideas as to the substitute feeding of their patients.

Emanating from Boston, laboratories have been established in this country in New York, Philadelphia, Chicago, Baltimore, Buffalo, Cincinnati, Cleveland, Gran in Rapids, Pittsburg, St. Louis and Washington; in Canada in Montreal, Ottawa and Toronto, and England in London. In connection with these labor tories are farms belonging to the Walker-Gordon Com pany, and especially equipped for supplying the best millo for substitute feeding. The farms and the laboratori 